\title{
CARACTERIZACION DE SINGULARIDADES DICRITICAS EN FOLIACIONES DE DIMENSION UNO
}

\author{
RENATO MARIO BENAZIC TOME \\ UNIVERSIDAD NACIONAL MAYOR DE SAN MARCOS
}

\section{INTRODUCCION}

En el presente trabajo, caracterizamos la presencia de singularidades dicríticas aisladas en foliaciones holomorfas de dimensión uno, en función del primer jet no nulo del campo vectorial holomorfo que define la foliación.

Sea $\mathscr{M}^{n}$ una variedad compleja de dimensión $n$ (el lector interesado en conocer detalles de la teoría de variedades analíticas y de las funciones de varias variables complejas, podrá consultar (6) y (8)) y consideremos en ella una foliación holomorfa singular de dimensión uno. Con esto queremos decir que en un punto $p \in \mathscr{M}^{n}$, la foliación es generada por el campo vectorial

$$
Z=\sum_{i=1}^{n} A_{i} \frac{\partial}{\partial . z_{i}}, \quad A_{i} \in O_{n, p}, \quad 1 \leq i \leq n, \operatorname{m.c.d} .\left(A_{1}, \ldots, A_{n}\right)=1
$$

donde $O_{n, p}$ es el anillo de los gérmenes de las funciones analíticas en $\boldsymbol{p}$. En lo que sigue, denotaremos a esta foliación por $F_{Z}$. y las funciones $\boldsymbol{A}_{\boldsymbol{i}}$ serán llamadas componentes de $Z$.

Sea $(U, \phi)$ una carta de $\mathcal{M}^{n}$ alrededor del punto $p \in \mathcal{M}^{n}$ tal que $(p)=$ 0 , luego $A_{i} \phi^{-1}$ es una función holomorfa de varias variables complejas en una vecindad del origen y por lo tanto ella tiene un desarrollo en series de potencias

$$
A_{i} \phi^{-1}=\sum_{k \geq 0} A_{i}^{\prime},: \leq i \leq n
$$

donde $\operatorname{los} A_{i}^{k}$ son polinomios homogéneos de grado $k$ de $\boldsymbol{n}$ variables -complejas. El orden de $A_{i} \phi^{-1}$ en 0 es por definición, el mínimo número entero $v_{i}$ tal que $A_{i}^{k} \equiv 0 \forall k<v_{i}$ y $A_{i}^{v} \neq 0$. Se demuestra que el número $v_{i}$ es independiente de la elección de la carta $(U, \phi)$, por ésta razón denotaremos $v_{i}=\operatorname{ord}_{p}\left(A_{i}\right)$. I a multiplicidad algebraica $m_{p}\left(F_{Z}\right)\left(\right.$ o $\left.m_{p}(Z)\right)$, de $\boldsymbol{F}_{Z}$ en el 
punto $p \in \mathcal{M}^{n}$, es el mínimo de los ordenes $\operatorname{ord}_{p}\left(A_{j}\right)$. Diremos que $p$ es un punto singular de $F_{Z}$ si $m_{p}(Z) \geq I$, caso contrario, decimos que $p$ es un punto regular. El conjunto de todos los punto singulares de la foliación será denotado por $\operatorname{Sing}\left(T_{Z}\right)$.

Sea $E: \mathcal{N}^{n} \rightarrow \mathcal{M}^{n}$ el blow-up con centro en el punto $p \in \operatorname{Sing}\left(F_{Z}\right)$, entonces existe una única manera de extender el pull-back $E^{*}\left(F_{Z}-\{p\}\right)$ a una foliación holomorfa singular $F_{Z}{ }^{*}$ en una vecindad del espacio proyectivo $C P(n-1)=E^{-1}(p) \subset \mathcal{N}^{n}$ con conjunto singular de codimensión $\geq 2$. En este caso, decimos que $F_{Z}{ }^{*}$ es la transformada estricta de $F_{Z}$ por $E$. El punto $p$ es una singularidad no-dicrítica de $F_{Z}$, cuando $E^{-1}(p)$ es invariante por $F_{Z}{ }^{*}$,esto significa que el espacio proyectivo es unión de hojas y singularidades de $F_{z}{ }^{*}$. Caso contrario, el punto $p$ es llamado singularidad dicrítica. El conjunto de las foliaciones holomorfas de dimensión uno en $\mathscr{M}^{n}$ con una singularidad dicrítica aislada, será denotado por $\mathcal{D}^{n}$. El resultado principal de este trabajo, establece que la presencia de una singularidad dicrítica aislada queda detectada por la forma del primer jet no nulo del campo $Z$.

\section{BLOW-UP EN UN PUNTO}

Este concepto es bien conocido y existe mucha literatura al respecto, lo que presentamos en esta sección es un breve resumen de los principales conceptos y propiedades. El lector interesado podrá consultar (2), (3), (5) y (7) para mayores detalles. El transformado $\left(C^{n}\right)^{*}$ de $C^{n}$ por blow-up en el punto 0 es la adherencia en $C^{n} \times C P(n-1)$, del gráfico $\mathrm{G}$ de la aplicación de paso al cociente:

$$
C^{n}-\{0\} \rightarrow C P(n-1) ; \quad z \rightarrow[z]
$$

Ella es una variedad holomorfa : si $y^{(j)}: U_{j}{ }^{*} \rightarrow C^{n-1}$ designa las cartas canónicas del espacio proyectivo, $y^{(j)}=\left(y_{1}^{j}, \ldots, y_{j-1}^{j}, y_{j+1}^{j}, \ldots, y_{n}^{\prime}\right), \quad y_{k}^{\prime}=\frac{z_{k}}{z_{j}}$, las aplicaciones:

$$
\left(z_{j}, y^{(j)}\right):\left(C^{n}\right)^{*} \cap\left(C \times U_{j}\right) \rightarrow C \times C^{n-1}
$$

Constituye un atlas de $\left(C^{n}\right)^{*}$. En estas cartas, la restricción $E:\left(C^{n}\right)^{*}$ $\rightarrow C^{n}$ de la primera proyección, se llama aplicación de blow-up y su expresión en coordenadas, es: 


$$
E\left(z_{j}, y^{(j)}\right)=\left(z, y_{1}^{\prime}, \ldots, z, y_{1-1}^{\prime}, z_{1}, z, y_{1+1}^{\prime}, \ldots, z, y_{11}^{\prime}\right)
$$

El divisor excepcional $E^{-1}(0)$ se identifica a $C P(n-1)$ y claramente $E$ es un isomorfismo de $\left(C^{n}\right)^{*}-C P(n-1)$ sobre $C^{n}-\{0\}$.

\section{SINGULARIDADES DICRITICAS EN DIMENSION DOS}

Considérese $Z\left(z_{1}, z_{2}\right)=A_{l}\left(z_{1}, z_{2}\right) \frac{\partial}{\partial \cdot z_{1}}+A_{2}\left(z_{1}, z_{2}\right) \frac{\partial}{\partial \cdot z_{2}}$ un campo vectorial holomorfo definido en una vecindad $U$ del $0 \in C^{2}$, el cual es un punto singular de $Z$. La ecuación diferencial ordinaria generada por $Z$ en $U$ es

$$
\begin{aligned}
& \frac{d z_{1}}{d T}=A_{1}\left(z_{l}, z_{2}\right) \\
& \frac{d z_{2}}{d T}=A_{2}\left(z_{1}, z_{2}\right)
\end{aligned}
$$

Suponiendo que $m_{0}(Z)=v$, tenemos

$$
\begin{aligned}
& \frac{d z_{1}}{d T}=A_{1}^{v}\left(z_{1}, z_{2}\right)+A_{1}^{v+1}\left(z_{1}, z_{2}\right)+\ldots \ldots \\
& \frac{d z_{1}}{d T}=A_{2}^{v}\left(z_{1}, z_{2}\right)+A_{2}^{v+1}\left(z_{1}, z_{2}\right)+\ldots \ldots
\end{aligned}
$$

Sea $U_{l}=\left\{\left(z_{1}, z_{2}\right) \in U: z_{1} \neq 0\right\}$ y $V_{1}=E^{-1}\left(U_{1}\right)$, donde $E$ es el blow-up centrado en el origen de $C^{2}$. En $v_{1}$ introducimos coordenadas $\left(y_{1}, y_{2}\right)$ y en esta carta, $E$ se expresa como $E\left(y_{1}, y_{2}\right)=\left(y_{1}, y_{1} y_{2}\right), y$ el pull-back de Z por $E$ es

$$
E^{*} Z=A_{1} E \frac{\partial}{\partial \cdot y_{1}}+\frac{A_{2} E-y_{2} A_{1} E}{y_{1}} \frac{\partial}{\partial \cdot y_{2}}
$$

de donde

$A_{1} E\left(y_{1}, y_{2}\right)=y_{1}^{v} A_{1}^{v}\left(1, y_{2}\right)+y_{1}^{v+1} A_{1}^{v+1}\left(1, y_{2}\right)+\ldots$

$\frac{A_{2} E-y_{2} A_{1} E}{y_{1}}\left(y_{1}, y_{2}\right)=y_{1}^{v-1}\left[A_{2}^{v}\left(1, y_{2}\right)-y_{2} A_{1}^{v}\left(1, y_{2}\right)\right]+y_{1}^{v}\left[A_{2}^{v+1}\left(1, y_{2}\right)-y_{2} A_{1}^{v+1}\left(1, y_{2}\right)\right]+\ldots$

Definiendo el polinomio homogéneo de grado $v+1$ : 


$$
P_{1,1}\left(z_{1}, z_{2}\right)=z_{1} A_{2}^{\prime}\left(z_{1}, z_{2}\right)+z_{2} A_{1}^{\prime \prime}\left(z_{1}, z_{2}\right)
$$

se observa que se presentan dos casos:

Caso 1: $P_{v+1}\left(z_{1}, z_{2}\right) \equiv 0$. Podemos dividir por $y_{1}^{\prime \prime}$ y considerar el campo vectorial $\frac{E^{*} Z}{y_{1}^{*}}$ el cual induce en $v_{1}$ la ecuación diferencial ordinaria

$$
\begin{aligned}
& \frac{d y_{1}^{\prime}}{d T}=A_{1}^{\prime}\left(1, y_{2}\right)+y_{1}^{\prime}\left[A_{1}^{\cdots+1}\left(1, y_{2}\right)+\ldots . .\right] \\
& \frac{d y_{2}}{d T}=A_{2}^{n+1}\left(1, y_{2}\right)-y_{2} A_{1}^{\prime+1}\left(1, y_{2}\right)+y_{2}\left[A_{2}^{w+2}\left(1, y_{2}\right)-y_{2} A_{1}^{\prime+2}\left(1, y_{2}\right)+\ldots . .\right]
\end{aligned}
$$

Se deduce inmediatamente que la foliación en $v_{1}$ tiene singularidades aisladas en los ceros comunes de los polinomios $A_{1}^{\prime \prime}\left(1, y_{2}\right)=$ 0 y $A_{2}^{\prime \prime 1}\left(1, y_{2}\right)-y_{2} A_{1}^{\prime \prime+1}\left(1, y_{2}\right)=0$. Además ésta foliación es transversal a la recta proyectiva $y_{1}=0$, en todos los puntos en donde $A_{1}^{*}\left(1, y_{2}\right) \neq 0$. Trabajando en la segunda carta del blow-up, se llega a resultados similares. Cuando se presenta éste caso, decimos que $0 \in C^{2}$ es una singularidad dicritica.

Caso 2: $P_{v+1}\left(z_{1}, z_{2}\right) \neq 0$ Ahora solo podemos dividir por $y_{1}^{v-1}$ y considerar el campo vectorial $\frac{E^{*} Z}{y_{1}^{v-1}}$ el cual induce en $V_{1}$ la ecuación diferencial ordinaria

$$
\begin{aligned}
& \frac{d y_{1}}{d T}=y_{1}\left[A_{1}^{v}\left(1, y_{2}\right)+\ldots\right] \\
& \frac{d y_{2}}{d T}=P_{v+1}\left(1, y_{2}\right)+y_{1}\left[A_{2}^{v+1}\left(1, y_{2}\right)+A_{1}^{v+1}\left(1, y_{2}\right)+\ldots .\right]
\end{aligned}
$$

También en este caso, la foliación inducida en $v_{1}$ tiene singularidades

aisladas, en los puntos $\left(0, y_{2}\right)$, en donde los $y_{2}$ son raices del polinomio $\mathrm{P}_{v+1}\left(1, \mathrm{y}_{2}\right)$. Observe que la recta proyectiva $y_{1}=0$ menos las singularidades es una hoja de la foliación.

Los detalles de los resultados obtenidos en la presente sección, el lector los podrá encontrar en (3) y (4) 


\section{EL RESULTADO PRINCIPAL}

A continuación nosotros generalizaremos los resultados obtenidos en la sección anterior a campos vectoriales holomorfos en dimensión arbitraria (finita). Sea $\mathcal{M}^{n}$ una variedad compleja $n$-dimensional $y$ consideremos una foliación holomorfa de dimensión uno $F_{\mathrm{Z}}$ sobre $\mathcal{M}^{\mathrm{n}}$. Supongamos que $p \in \mathcal{M}^{n}$

sea una singularidad aislada de $F_{z}$. Denotemos por $z=\left(z_{1}, \ldots, z_{n}\right)$ las coordenadas locales de una vecindad de $p$ en $\mathcal{M}^{n}$ tal que $p=(0, \ldots, 0) \in C^{n}$. En estas condiciones, $F_{Z}$ es generado por el campo vectorial holomorfo

$$
Z=\sum_{i=1}^{n} A_{i} \frac{\partial}{\partial \cdot z_{i}}
$$

Si $m_{o}(Z)=v$, entonces las componentes $A_{i}$ de $Z$ tienen un desarrollo de Taylor en $0 \in C^{n}$

$$
A_{i}=\sum_{k \geq v} A_{k}^{\prime}, \quad 1 \leq i \leq n
$$

donde los $A_{k}^{\prime}$ son polinomios homogéneos de grado $k$.Este campo vectorial induce, sobre una vecindad del $0 \in C^{n}$ el siguiente sistema de ecuaciones diferenciales ordinarias:

$$
\begin{aligned}
& \frac{d z_{1}}{d T}=\sum_{k \geq v} A_{k}^{1}\left(z_{1}, z_{2}, \ldots, z_{n}\right) \\
& \frac{d z_{2}}{d T}=\sum_{k \geq v} A_{k}^{2}\left(z_{1}, z_{2}, \ldots, z_{n}\right) \\
& \frac{d z_{n}}{d T}=\sum_{k \geq v} A_{k}^{n}\left(z_{1}, z_{2}, \ldots, z_{n}\right)
\end{aligned}
$$

En el siguiente Teorema, probamos que la condición de que $p$ sea una singularidad dicrítica de $F_{\mathrm{Z}}$ puede ser caracterizada en términos de los polinomios $A_{1}^{v}(1 \leq i \leq n)$, i.e. de $J_{0}^{v}(Z)$ : el Jet de orden $v$ de $Z$ en el origen. 
Teorema.- Con las notaciones anteriores, las siguientes condiciones son equivalentes:

a) $F_{\mathrm{Z}} \in \mathcal{D}^{n}$.

b) $z_{1} A_{v}^{\prime}-z_{1} A_{v}^{\prime}=0 ; \forall 1 \leq i<j \leq n$.

c) $\exists P_{v-1}$ polinomio homogéneo de grado $v-1$ tal que $A_{v}^{\prime}=z_{1} P_{v-1}, \forall 1 \leq i \leq n$.

d) $J_{0}^{r}(Z)=P_{v_{-1}} R$, donde $R=\sum_{i=1}^{n} z_{1} \frac{\partial}{\partial \cdot z_{1}}$ es el campo radial.

\section{Prueba:}

a) $\Rightarrow$ b) Para cada $j=1, \ldots, n$ sea $U_{j}=\left\{\left(z_{1}, \ldots, z_{n}\right) \in C^{n}: z_{j} \neq 0\right\}$ y $U_{j}{ }^{*}=E^{-1}\left(U_{\mathrm{j}}\right)$, donde $\mathrm{E}$ es el blow-up centrado en $0 \in \mathrm{C}^{\mathrm{n}}$. En $U_{j}{ }^{*}$ introducimos coordenadas

$\left(y_{1}, \ldots, y_{n}\right)$, de esta manera $E$ tiene la siguiente expresión:

$$
E\left(y_{1}, \ldots, y_{n}\right)=\left(z_{1}, \ldots, z_{n}\right) ; \text { donde } z_{i}=y_{y_{j}} \text { si } i \neq j \text { y } z_{j}=y_{j}
$$

Además:

$$
E^{-1}(0) \cap U_{j}^{*}=:\left\{\left(y_{1}, \ldots, y_{n}\right) \in U_{j}^{*}: y_{j}=0\right\}
$$

En esta carta, el pull-back de $Z$ por $E$ es generado por:

$$
E^{*} Z=A_{j} E \frac{\partial}{\partial \cdot y_{j}}+\sum_{i=1, i * j}^{n}\left(\frac{A_{i} E-y_{1} A_{j} E}{y_{j}}\right) \frac{\partial}{\partial \cdot y_{i}}
$$

De (1):

Por (4)

$$
A_{i} E(y)=\sum_{k \geq v} y_{j}^{k} A_{k}^{i}\left(y^{*}\right), \text { donde } y=\left(y_{1}, \ldots, y_{n}\right), y^{*}=\left(y_{1}, \ldots, y_{j-1}, 1, y_{j+1}, \ldots y_{n}\right)
$$

$$
E^{*} Z(y)=\left(\sum_{k \geq v} y_{j}^{k} A_{k}^{j}\left(y^{*}\right)\right) \frac{\partial}{\partial \cdot y_{j}}+\sum_{i=1, i \neq j}^{n}\left(\sum_{k \geq v} y_{j}^{k-1}\left[A_{k}^{\prime}\left(y^{*}\right)-y_{i} A_{k}^{\prime}\left(y^{*}\right)\right]\right) \frac{\partial}{\partial \cdot y_{i}}
$$

Suponga por contradicción que b) es falso, entonces existe $i \neq j$, $1 \leq i \leq n$, tal que $A_{v}^{\prime}\left(y^{*}\right)-y_{i} A_{\nu}^{j}\left(y^{*}\right) \neq 0$, por lo tanto, $E^{*} Z$ es divisible por $y_{j}^{\nu-1}$. Sea $Z^{*}=\frac{E^{*} Z}{y_{J}^{v-1}}$, claramente $F_{Z}^{*}$ es la foliación generada por $Z^{*}$ sobre una vecindad de $E^{-1}(0) \cap U_{j}^{*}$. De (5) tenemos que: 


$$
Z^{*}(y)=y_{1} A_{v}^{\prime}\left(y^{*}\right) \frac{\partial}{\partial \cdot y_{1}}+\sum_{i=1,1 * j}^{n}\left(A_{v}^{\prime}\left(y^{*}\right)-y_{1} A_{v}^{\prime}\left(y^{*}\right)\right) \frac{\partial}{\partial y_{1}}+y_{1} Y^{*}(y)
$$

donde $Y^{*}$ es un campo vectorial holomorfo. De (6), se deduce fácilmente que $E^{\prime}(0)$ es invariante por $F_{Z}{ }^{*}$, lo cual es una contradicción, desde que $F_{Z}$ $\in \mathcal{D}^{n}$.

b) $\Rightarrow c)$ Consideremos $A_{v}^{\prime}\left(z_{1}, \ldots z_{n}\right)=\sum_{k=0}^{v} a_{v-k}^{\prime}\left(z_{2}, \ldots, z_{n}\right) z_{1}^{k}$, donde $a_{v-k}^{\prime} \cdot(1 \leq i \leq n)$ son polinomios homogéneos de grado $v-k$ en las variables $z_{1}, \ldots, z_{n}$. De las hipótesis, deducimos que:

$0=z_{j} A_{v}^{\prime}-z_{1} A_{v}^{\prime}=\sum_{k=0}^{v} a_{v-k}^{J} z_{j} z_{1}^{k}-\sum_{k=0}^{v} a_{v-k}^{\prime} z_{J}^{k+1}=a_{v}^{\prime} z_{j}+\sum_{k=1}^{v}\left(a_{v-k}^{\prime} z_{\jmath}-a_{v-k+1}^{\prime}\right) z_{1}^{k}-a_{0}^{\prime} z_{1}^{v+1}$

donde $1<j \leq n$. Por tanto:

$$
\begin{aligned}
& a_{v,}^{1}=0 \\
& a_{v-k}^{\prime}=z, a_{v-k-1}^{1}, 0 \leq k \leq v-1 \\
& a_{0}^{\prime}=0
\end{aligned}
$$

y por tanto:

$$
\begin{gathered}
A_{v}^{\prime}=\sum_{k=1}^{v} a_{v-k}^{1} z_{1}^{k}=z_{1}\left(\sum_{k=0}^{v-1} a_{v-k-1}^{1} z_{1}^{k}\right) \\
A_{v}^{\prime}=\sum_{k=0}^{v-1} a_{v-k}^{j} z_{1}^{k}=\sum_{k=0}^{v-1} a_{v-k-1}^{1} z_{j} z_{1}^{k}=z_{J}\left(\sum_{k=0}^{v-1} a_{v-k-1}^{1} z_{1}^{k}\right), 1 \leq j \leq n .
\end{gathered}
$$

Deducimos de aquí que $P_{v-1}\left(z_{1}, \ldots, z_{n}\right)=\sum_{k=0}^{v-1} a_{v-k-1}^{1}\left(z_{2}, \ldots, z_{n}\right) z_{1}^{k}$ es el polinomio homogéneo de grado $v-1$ buscado.

c) $\Rightarrow$ d) $J_{0}^{v}(Z)=\sum_{i=1}^{n} A_{v}^{i} \frac{\partial}{\partial \cdot z_{i}}=\sum_{i=1}^{n} z_{i} P_{v-1} \frac{\partial}{\partial \cdot z_{i}}=P_{v-1} \sum_{i=1}^{n} z_{i} \frac{\partial}{\partial \cdot z_{i}}$.

d) $\Rightarrow$ a) Por hipótesis y (5), tenemos: 
$E^{*} Z(y)=y_{1}^{v}\left(P_{v-1}\left(y^{*}\right) \frac{\partial}{\partial y_{1}}+\sum_{i=1, i+j_{j}}^{n}\left(A_{v+1}^{\prime}\left(y^{*}\right)-y_{1} A_{v+1}^{\prime}\left(y^{*}\right)\right) \frac{\partial}{\partial \cdot y_{1}}\right)+y_{1}^{v+1} Y^{*}(y)$.

se sigue que $E^{*} Z$ es divisible por $y_{j}^{v}$ y $F_{Z}{ }^{*}$ es la foliación generada por $Z^{*}=\frac{E^{*} Z}{y_{1}^{*}}$ donde:

$$
Z(y)=\left(P_{v-1}\left(y^{*}\right) \frac{\partial}{\partial \cdot y_{1}}+\sum_{i=1,1,1=1}^{n}\left(A_{v+1}^{\prime}\left(y^{*}\right)-y_{1} A_{v+1}^{\prime}\left(y^{*}\right)\right) \frac{\partial}{\partial \cdot y_{1}}\right)+y_{1} Y^{*}(y) .
$$

en donde $Y^{*}$ es un campo vectorial holomorfo. Por (3), tenemos que:

$$
Z^{*}=\left(P_{v-1}\left(y^{*}\right) \frac{\partial}{\partial \cdot y_{j}}+\sum_{i=1,1, \infty}^{n}\left(A_{v+1}^{\prime}\left(y^{*}\right)-y_{1} A_{v+1}^{\prime}\left(y^{*}\right)\right) \frac{\partial}{\partial \cdot y_{1}}\right) .
$$

Esto implica que $E^{\prime}(0)$ no es invariante por $F_{Z}{ }^{*}$ y por tanto $F_{Z} \in \mathscr{D}^{n}$, lo cual finaliza la demostración.

Considere una foliación $F_{Z} \in \mathscr{D}^{n}$. De (8) observamos que los puntos de $\operatorname{Sing}\left(F_{Z^{*}}\right) \cap E^{\prime}(0)$ en la carta $U_{j}^{*}(j$ fijo, $1 \leq j \leq n)$ son las soluciones de:

$$
\begin{aligned}
& P_{v-1}\left(y^{*}\right)=0 \\
& A_{v+1}^{\prime}\left(y^{*}\right)-y_{1} A_{v+1}^{\prime}\left(y^{*}\right)=0 ; \quad 1 \leq i \leq n, i \neq j
\end{aligned}
$$

Concluimos que $\operatorname{Sing}\left(F \mathrm{z}^{*}\right) \cap E^{\prime}(0)$ es el conjunto de puntos $\left[z_{1} ; \ldots ; z_{n}\right] \in C P(n-1)$ las cuales son soluciones de las siguientes $\frac{(n-1) n}{2}+1$ sistema de ecuaciones homogéneas:

$$
\begin{aligned}
& P_{v-1}(z)=0 \\
& z_{,} A_{v+1}^{\prime}(z)-z_{1} A_{v+1}^{j}(z)=0 ; 1 \leq i<j \leq n
\end{aligned}
$$

De ésta manera, la prueba del siguiente Corolario, es evidente:

Corolario.- Con las notaciones anteriores, tenemos que:

$F_{\mathrm{Z}} \in D_{0}^{n}$ si y solo si $z=0 \in C^{n}$ es la única solución del sistema (9). 
Finalmente, observe que para cada $F_{Z} \in D_{0}^{\prime \prime}$, definimos

$$
S=\left\{\left[z_{1} ; \ldots ; z_{n}\right] \in C P(n-1): P_{v-1}\left(z_{1}, \ldots, z_{n}\right)=0\right\}
$$

Claramente, $\mathrm{S}$ es una hipersuperfície algebraica en $C P(n-1)$ (ver (1)) y tiene la siguiente propiedad : Sea $p^{*} \in E^{-1}(0)$ y $L^{*}$ la hoja de $F_{Z^{*}}$ que pasa por $p^{*}$. Se sigue inmediatamente que $L^{*}$ es no singular y si $p^{*} \notin S$ entonces $L^{*}$ es transversal al espacio proyectivo $E^{-1}(0)$. Por esta razón llamaremos a $S$ la hipersuperficie de tangencia de la foliación $F_{Z}$.

\section{REFERENCIAS}

(1) BENAZIC, R. Isolated dicritical singularities of a holomorphic vector fields, Tesis de doctorado, IMPA, (1996).

(2) CAMACHO, C. - LINS NETO, A. - SAD, P. Topological invariants and Equidesingularization for Holomorphic Vector Fields, Journal of differential geometry, 20,(1984), $143-174$

(3) CAMACHO, C. Holomorphic Dynamical Systems, Summer School on Dynamical Systems, ICTP, (19889.

(4) CAMACHO, C. - SAD, P. Pontos singulares de Equaçoes Diferenciais Analiticas, $16^{\circ}$ Colóquio Brasileiro de Matemáticas, IMPA, (1987).

(5) CERVEAU, D. - MATTEI, J-F. Formes Intégrables Holomorphes Singulières, astérisque, 97, (1982).

(6) GUNNING, R. - ROSSI, H. Analytic functions of several complex variables. Prentice-Hall, Englewood Cliffs, NJ. (1965)

(7) MATTEI, J-F. - MOUSSU, R. Holonomie et intégrales premières, Ann. Sci. Ecole. Norm. Sup. (4), 13, (1980), 469 - 523

(8) WHITNEY, H. Complex Analytic Varieties, Addison-Wesley Publishing Compary, (1972). 